\title{
Human IgM and IgG Responses to an Inactivated SARS-CoV-2 Vaccine*
}

\author{
Jo-Lewis BANGA NDZOUBOUKOU ${ }^{1}$, Yan-di ZHANG ${ }^{1}$, Qing LEI ${ }^{1}$, Xiao-song LIN ${ }^{1}$, Zong-jie YAO ${ }^{1}$, Hui FU ${ }^{1}$, Le-yong \\ YUAN $^{2,3 \#}$, Xiong-lin FAN ${ }^{1 \#}$ \\ ${ }^{1}$ Department of Pathogen Biology, School of Basic Medicine, Tongji Medical College, Huazhong University of Science and \\ Technology, Wuhan 430030, China \\ ${ }^{2}$ Department of Clinical Laboratory, Southern University of Science and Technology Hospital, Shenzhen 518055, China \\ ${ }^{3}$ Hubei Key Laboratory of Wudang Local Chinese Medicine Research, Hubei University of Medicine, Shiyan 442000, China
}

(C) Huazhong University of Science and Technology 2021

\begin{abstract}
[Abstract] Objective: The ongoing COVID-19 pandemic warrants accelerated efforts to test vaccine candidates. To explore the influencing factors on vaccine-induced effects, antibody responses to an inactivated SARS-CoV-2 vaccine in healthy individuals who were not previously infected by COVID-19 were assessed. Methods: All subjects aged 18-60 years who did not have SARS-CoV-2 infection at the time of screening from June 19, 2021, to July 02, 2021, were approached for inclusion. All participants received two doses of inactivated SARS-CoV-2 vaccine. Serum IgM and IgG antibodies were detected using a commercial kit after the second dose of vaccination. A positive result was defined as $10 \mathrm{AU} / \mathrm{mL}$ or more and a negative result as less than $10 \mathrm{AU} / \mathrm{mL}$. This retrospective study included 97 infection-naïve individuals (mean age 35.6 years; $37.1 \%$ male, $62.9 \%$ female). Results: The seropositive rates of IgM and IgG antibody responses elicited after the second dose of inactivated SARS-CoV-2 vaccine were $3.1 \%$ and $74.2 \%$, respectively. IgG antibody levels were significantly higher than IgM levels $(P<0.0001)$. Sex had no effect on IgM and IgG antibody response after the second dose. The mean anti-IgG level in older persons ( $\geqslant 42$ years) was significantly lower than that of younger recipients. There was a significantly lower antibody level at $>42$ days compared to that at $0-20$ days $(P<0.05)$ and $21-31$ days $(P<0.05)$ after the second dose. Conclusion: IgG antibody response could be induced by inactivated SARS-CoV-2 vaccine in healthy individuals ( $>18$ years), which can be influenced by age and detection time after the second dose of vaccination.
\end{abstract}

Key words: COVID-19; inactivated SARS-CoV-2 vaccine; IgM and IgG antibody responses

The Coronavirus disease 2019 (COVID-19) is caused by severe acute respiratory syndrome coronavirus 2 (SARS-CoV-2) ${ }^{[1-5]}$. From the emergence of COVID-19 at the end of 2019 to September 2021, more than 110 million cases and more than 4 million deaths had been recorded, indicating that COVID-19 poses a substantial threat to public health worldwide ${ }^{[6]}$. The pandemic has significantly affected people's lives with substantial disruption of health services and

Jo-Lewis BANGA NDZOUBOUKOU, E-mail: bangas19@ yahoo.fr

\#Corresponding authors, Xiong-lin FAN, E-mail: xlfan@, hust.edu.cn; Le-yong YUAN, E-mail: leyongyuan@whu. edu.cn

${ }^{*}$ This project was supported by grants from the Applied Basic Research Key Project of Wuhan Municipal Bureau of Science and Technology (No. 2020020601012218), the Fundamental Research Funds for the Central Universities (HUST COVID-19 Rapid Response Call No. 2020kfyXGYJ040), and National Natural Science Foundation of China (No. 81802090). considerable impact on the global economy. Because of the highly contagious nature of SARS-CoV-2 and the severe clinical outcomes ${ }^{[7,8]}$, one of the primary strategies to control the pandemic is to develop an effective vaccine. As such, clinical trials of several vaccines have been initiated $^{[0-12]}$. To date, 184 and 112 vaccine candidates to prevent against COVID-19 are in the pre-clinical and clinical development phases, respectively ${ }^{[13]}$. Currently, the most promising hope for ending this pandemic is the successful rollout of widespread COVID-19 vaccination. At least 17 vaccines (inactivated, viral vector, protein subunit, or mRNA) have received emergency use authorization for immunization of health-care workers and at-risk individuals ${ }^{[14-17]}$. Some reports suggest that mixing and matching COVID-19 vaccines might produce a robust immune response ${ }^{[18]}$. However, the influencing factors on the immunogenicity induced by the inactivated SARS-CoV-2 vaccine have not been fully explored. To this end, the present study assessed antibody responses to the inactivated SARS-CoV-2 vaccine among vaccine recipients in Shenzhen, China. 


\section{MATERIALS AND METHODS}

\subsection{Study Design and Participants}

This was a retrospective study involving participants between the ages of 18-60 years (table 1), who did not have SARS-CoV-2 infection, confirmed by negative results of serum specific IgM and IgG antibodies using a commercial SARS-CoV-2 rapid test kit (Innovita, China) and negative nucleic acid results using pharyngeal swabs or sputum and anal swabs with a nucleic acid diagnostic kit (Liferiver, China) at the time of screening. All participants received the two recommended doses of an inactivated SARS$\mathrm{CoV}-2$ vaccine. The vaccine was developed by the Beijing Institute of Biotechnology (China) or Sinovac (China). We excluded vaccine recipients who had any of the following conditions: a history of travelling to regions outside of China or regions with reported COVID-19 cases from December 2019; a history of household contact with the confirmed infection with SARS-CoV-2; symptoms such as fever, cough, runny nose, sore throat, diarrhea, dyspnea, or tachypnoea before examination for serum antibodies; and rheumatic immune diseases including systemic lupus erythematosus and rheumatoid arthritis.

Table 1 Characteristics of study participants $(n=97)$

\begin{tabular}{lcc}
\hline Characteristics & $n(\%)$ & Mean (SD) \\
\hline $\begin{array}{l}\text { Number of participants } \\
\text { Age groups (years) }\end{array}$ & 97 & $35.6(10.2)$ \\
$20-30$ & $37(38.2)$ & $26.2(2.5)$ \\
$31-41$ & $35(36.0)$ & $34.4(2.2)$ \\
$42-52$ & $15(15.5)$ & $47.2(2.7)$ \\
$\geq 53$ & $10(10.3)$ & $56.1(1.4)$ \\
Gender & & \\
Male & $36(37.1)$ & \\
Female & $61(62.9)$ & \\
\hline
\end{tabular}

SD, standard deviation; Data are in $n(\%)$.

\subsection{Assessment of Antibody Responses}

Serum antibodies against SARS-CoV-2 IgM and IgG were detected using YHLO-CLIA IgM and IgG kits (YHLO Biotech Co., Ltd., China) after the second dose of vaccination according to the manufacturer's instructions. This chemiluminescence immunoassay used recombinant antigens that contained the nucleoprotein and spike protein of SARS-CoV-2 as targets for IgM and IgG antibodies. A positive result was defined as $10 \mathrm{AU} / \mathrm{mL}$ or more and a negative result as less than $10 \mathrm{AU} / \mathrm{mL}$. The kit was previously shown to detect IgM and IgG with sensitivity of $83 \%$ and $93 \%$, respectively, and specificity of $99 \%$ and $92 \%$, respectively ${ }^{[19]}$.

\subsection{Ethical Consideration}

The study protocol was approved by the Ethics Committee of Southern University of Science and Technology Hospital, Shenzhen, China. Informed written consent was obtained from the participants before inclusion, and ethical principles conformed to the current Declaration of Helsinki. In addition, the study was conducted according to the ethical and legal requirements of the country.

\subsection{Statistical Analysis}

Participants who were $\leqslant 41$ years of age were defined as younger participants, and those $\geqslant 42$ years were defined as older. All statistical analyses and figure drawings were performed with Prism (GraphPad) version 8.3.1. Participants' demographic characteristics are expressed as proportions. In all evaluations, $P<0.05$ are considered statistically significant. The categorical variables are expressed as $n(\%)$.

\section{RESULTS}

Between June 19, 2021, and July 02, 2021, 97 participants were recruited and vaccinated with two doses of an inactivated SARS-CoV-2 vaccine. The mean age of the cohort was 35.6 years, and the age distribution of this cohort was as follows: $20-30$ years old, $37(38.2 \%)$; 31-41 years old, $34(35.0 \%)$; 42-52 years old, $15(15.5 \%)$, and $\geqslant 53$ years old, $10(10.3 \%)$. The cohort had a slightly greater representation of female subjects, with $62.9 \%$ female and $37.1 \%$ male subjects. The basic characteristics of the cohort are shown in table 1. In our cohort, the post-vaccination $\operatorname{IgG}$ antibody level response elicited by the second vaccine dose was $74.2 \%(n=72)$. However, only $3.1 \%$ $(n=3)$ participants developed an IgM response after the second dose of the vaccine (table 2). Our results showed that the IgG antibody level was significantly higher than the IgM level $(P<0.0001)$ after the second dose of vaccine (fig. 1). To explore the influencing factors on the vaccine-induced immunogenicity, the IgM and IgG antibody levels after the second vaccine dose were compared among the recipients with respect to age and sex. Among all vaccinated subjects, we found that sex (male and female) was not associated with anti-IgM and anti-IgG levels after receiving the second dose of inactivated SARS-CoV-2 vaccine (fig. 2A and 2B). Although there were no statistically significant differences in IgM and IgG antibody levels among different age groups, the mean anti-IgG level in the older groups ( $42-52$ years and $\geqslant 53$ years) was significantly lower than that of the younger group (fig. $3 \mathrm{~A}$ and $3 \mathrm{~B}$ ). More importantly, there was a significantly lower antibody level at $>42$ days compared to $0-20$ days $(P<0.05)$ and $21-31$ days $(P<0.05)$ after the second dose (fig. 4A and 4B).

\section{DISCUSSION}

Vaccination is an important strategy for controlling viral infections, especially when no approved drugs 


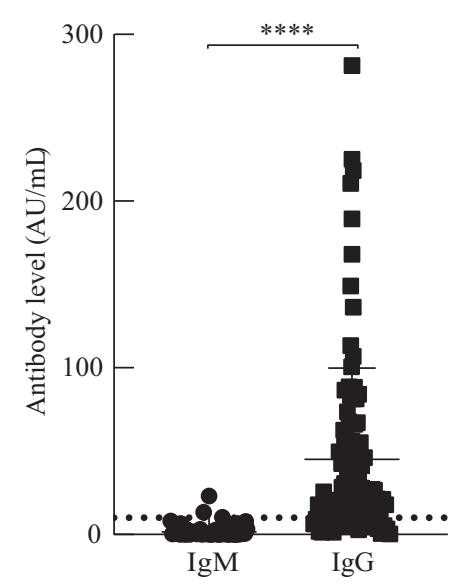

Fig. 1 Comparison of SARS-CoV-2 specific IgM and IgG antibody levels after the second dose of vaccine $(n=97)$. Dotted lines reflect limits of $\operatorname{IgG}$ and $\operatorname{IgM}$ detection. ${ }^{* * * *} P<0.0001$

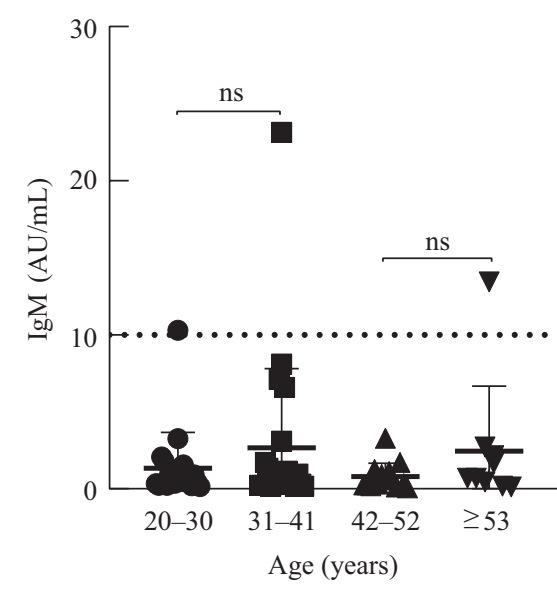

B
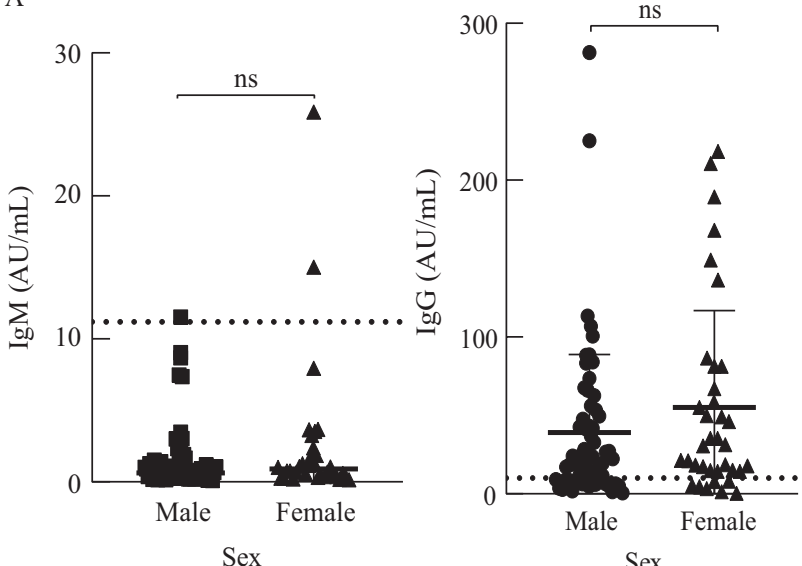

Fig. 2 Analysis of SARS-CoV-2 specific IgM (A) and IgG (B) antibody levels in subjects sub-grouped according to sex $(n=97)$. Dotted lines reflect limits of IgG and IgM detection. ns, non-statistically significant

B

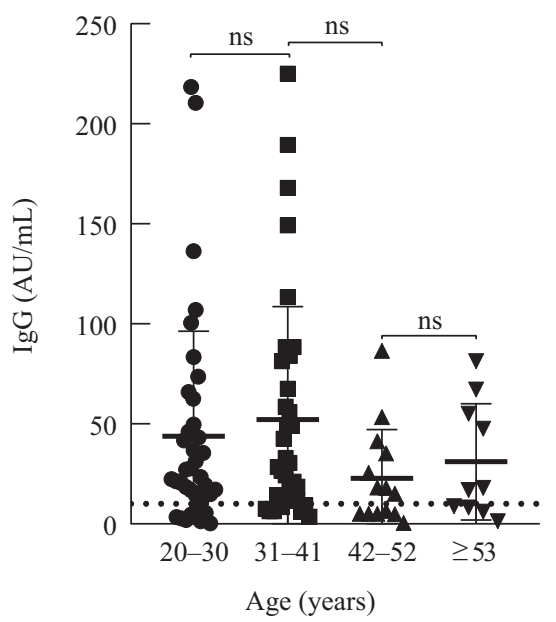

Fig. 3 Anti SARS-CoV-2 IgM (A) and IgG (B) levels in subjects sub-grouped by age ( $n=97)$. Dotted lines reflect limits of IgG and IgM detection. ns, non-statistically significant

A

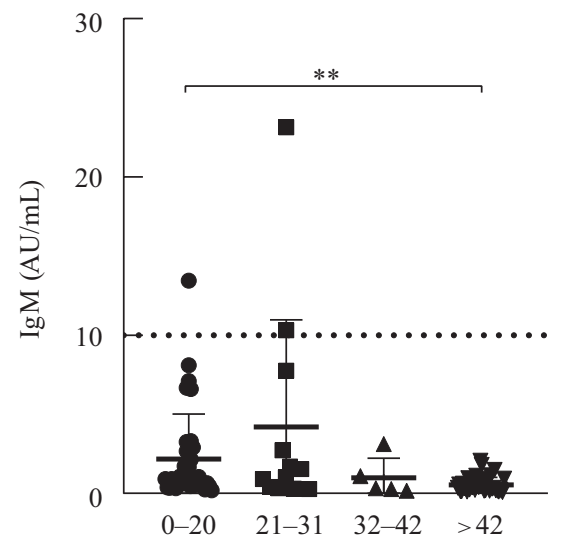

Interval time before antibodies detection (days)

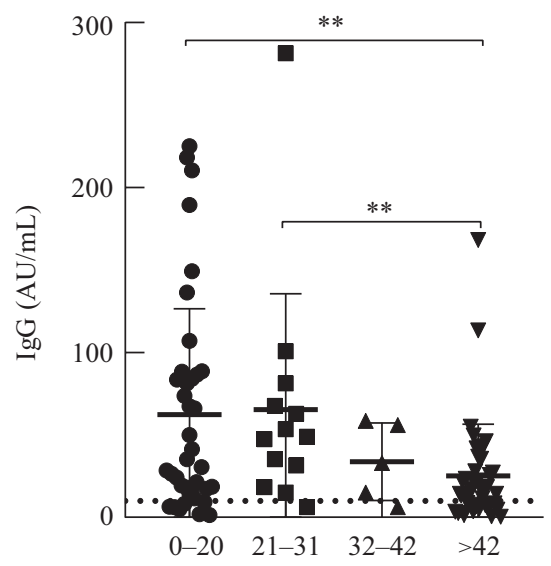

Interval time before antibodies detection (days)

Fig. 4 Evolution of SARS-CoV-2 IgM (A) and IgG (B) antibody levels per day after the second dose of vaccine ( $n=97)$. Dotted lines reflect limits of $\operatorname{IgG}$ and $\operatorname{IgM}$ detection. ${ }^{* *} P<0.05$ 
Table 2 Antibody responses of the vaccine recipient after the second dose of vaccination $(n=97)$

\begin{tabular}{lcc}
\hline Antibody responses & \multicolumn{2}{c}{ After the 2nd dose of vaccination } \\
\hline Responses categories & $\operatorname{IgM}$ & IgG \\
Negative $(<10 \mathrm{AU} / \mathrm{mL})$ & $94(96.9 \%)$ & $25(25.8 \%)$ \\
Positive $(\geq 10 \mathrm{AU} / \mathrm{mL})$ & $3(3.1 \%)$ & $72(74.2 \%)$ \\
\hline
\end{tabular}

IgM, immunoglobulin $\mathrm{M}$; IgG, immunoglobulin $\mathrm{G}$

exist to fight the emerging viruses. The COVID-19 pandemic is causing incessant disruption in people's activities and taking millions of lives worldwide. Despite the high efficacy of most currently approved vaccines, particularly the efficacy at limiting the risk of clinical aggravation ${ }^{[20]}$, accumulating data show considerable heterogeneity in the immune response elicited after vaccination among different individuals ${ }^{[21-25]}$. In this short retrospective study, we sought to evaluate the immunogenicity of an inactivated SARS-CoV-2 vaccine in producing an antibody response in the population who had not been infected SARS-CoV-2. We found that $74.2 \%$ of participants who received the second dose of the vaccine developed a significant $\operatorname{IgG}$ antibody response. At the same time, we also observed that a low percentage of participants who received the second dose of vaccine developed an IgM antibody response. We conclude that two vaccine doses with an inactivated SARS-CoV-2 vaccine can induce a humoral response in people $\geq 18$ years of age.

In particular, a study conducted in Chile showed that the vaccine efficacy of an inactivated SARSCoV-2 vaccine (CoronaVac) was around $65.9 \%$ for the prevention of COVID-19 infections ${ }^{[26]}$. In the present study, the comparison made between the IgM and $\operatorname{IgG}$ antibody responses induced in the vaccinated subjects shows that the IgG plays an essential role in the humoral responses directed against the SARS$\mathrm{CoV}-2$ virus. Indeed, it is known that IgG levels are crucial in ensuring protection against viral diseases ${ }^{[27]}$. In addition, IgG tends to be longer-lived and represent the class of antibodies predominantly evaluated in clinical and research settings for determining longterm protection or immunity ${ }^{[28]}$. Our result was similar to two recent studies conducted by Jalkanen et al. and Röltgen and colleagues. Jalkanen et al reported that in response to BNT162b2, subjects developed weak IgM antibody responses after two doses of the vaccine, in contrast to a strong $\operatorname{IgG}$ response ${ }^{[29]}$. Likewise, the report by Röltgen and colleagues showed that the BNT162b2 vaccine compared to natural infection induced moderate levels of anti-RBD IgM but did not induce a strong $\operatorname{IgG}$ response ${ }^{[30]}$. Thus, seropositive IgG antibody response after immunization might be an alternative biomarker to reflect vaccine efficacy.

On the other hand, the rapid development of several vaccines against SARS-CoV-2 represents a promising strategy to stop the spread of the infection and probably to reach a potential stage of herd immunity within the population ${ }^{[15,31,32]}$. According to a World Health Organization report, vaccination saves nearly 3 million lives every year by preparing the system's immune to fight off potential pathogens ${ }^{[33]}$. Apart from the individual protection acquired by vaccination, it also builds herd immunity, meaning immunization of a significant number of the population to protect vulnerable people (unvaccinated, immunologically naïve, and immunocompromised) by reducing the number of vulnerable people below the transmission threshold ${ }^{[34]}$. For example, a vaccination percentage of nearly $80 \%$ against the smallpox virus reduced transmission rates to uninfected people to levels so low that the virus can be eradicated ${ }^{[33]}$. Kwok et al. showed that the threshold value capable of inducing herd immunity against SARS-CoV-2 was approximately $67 \%{ }^{[35]}$. Herd immunity is an important weapon in the fight against epidemics, and vaccination is the way forward to achieve this immunity.

Our study showed that sex (male and female) was not associated with anti-IgM and anti-IgG levels among all vaccinated subjects after receiving the second dose of inactivated SARS-CoV-2 vaccine. Although there was no statistical difference in IgM and IgG antibody levels between different age groups, the mean anti-IgG level of the older groups (42-52 and $\geq 53$ years old) appeared significantly lower than that of the younger group. Our finding is consistent with another study, where the Euroimmun anti-SARS-CoV-2 S1 IgG ELISA assay was used to monitor humoral response to SARS-CoV-2 mRNA BNT162b2 vaccine. This study reported no statistically significant correlation between the age and sex of the individuals and the immune response caused by the vaccine ${ }^{[36]}$. However, Otavio et $a l^{[37]}$ observed in the elderly population a strong decrease in vaccine effectiveness with increasing age. Another report showed that older persons had significantly lower levels of antibodies than younger persons after measuring SARS-CoV-2 spike $\mathrm{S} 1 \mathrm{IgG}$ titers by ELISA in a small cohort consisting of 176 recipients ${ }^{[38]}$. According to the literature, age is an important factor in the humoral response induced after vaccination regardless of chronic medical conditions ${ }^{[24]}$. It has been demonstrated that people older than 60 years have an increased risk of severe illness and death from COVID-19, especially those with underlying chronic conditions. In fact, the response to vaccines is usually reduced in older adults due to immune senescence. Increasing sample size to analyze the effect of age on the immunogenicity of vaccine should be pursued.

Additionally, our results indicate that the antibody response, especially the IgG response of infectionnaïve vaccine recipients, was lower with increasing time after receiving the second vaccine dose. This decline in immune responses is often expected because 
a large number of plasmablasts (cells produced by B lymphocytes to fight a specific antigen) induced by the majority of vaccines are often not maintained as long-lived memory plasma cells ${ }^{[39-41]}$. Recently, Turner et al showed that in vaccinated people it was possible to observe high frequencies of plasmablasts in the draining lymph nodes and Spike-binding germinal center B cell 12 weeks after the second immunization ${ }^{[42]}$. Thus, carrying out longitudinal studies is essential to determine whether antibody levels will continue to decline or peak at a lower level.

Following the development of SARS-CoV-2 vaccines, some important questions should be addressed. The first concern is the durability of protection over a long period after vaccination and the second concern is determining the effect of a booster dose to prolong the duration of immunity against SARS-CoV-2 infection. Thus, to boost IgG response, the third dose of inactivated vaccine might still be needed for immunization.

In brief, an inactivated-SARS-CoV-2 vaccine can induce an anti-SARS-CoV-2 IgG antibody response in healthy individuals older than 18 , and the response might be influenced by age and the detection time after immunization.

\section{Conflict of Interest Statement}

We declare that we have no conflicts of interest.

\section{REFERENCES}

1 Chan JFW, Yuan S, Kok KH, et al. A familial cluster of pneumonia associated with the 2019 novel coronavirus indicating person-to-person transmission: a study of a family cluster. Lancet, 2020,395(10223):514-523

2 Chen N, Zhou M, Dong X, et al. Epidemiological and clinical characteristics of 99 cases of 2019 novel coronavirus pneumonia in Wuhan, China: a descriptive study. Lancet, 2020,395(10223):507-513

3 Li Q, Guan X, Wu P, et al. Early Transmission Dynamics in Wuhan, China, of Novel Coronavirus Infected Pneumonia. N Engl J Med, 2020,382(13):1199-1207

4 Wang C, Horby PW, Hayden FG, et al. A novel coronavirus outbreak of global health concern. Lancet, 2020,395(10223):470-473

5 Zhu N, Zhang D, Wang W, et al. A Novel Coronavirus from Patients with Pneumonia in China, 2019. N Engl J Med, 2020,382(8):727-733

6 Weekly epidemiological update on COVID-19 - 24 August 2021. Available from: https://www.who.int/ publications $/ \mathrm{m} /$ item/weekly-epidemiological-update-on COVID-19---24-august-2021. [Accessed August 29, 2021].

7 Tezer H, Bedir Demirdağ T. Novel coronavirus disease (COVID-19) in children. Turk J Med Sci, 2020,50(SI1):592-603

8 Cruz MP, Santos E, Velázquez Cervantes MA, et al. COVID-19, a worldwide public health emergency. Rev Clin Esp (Barc), 2021,221(1):55-61

9 Xia S, Duan K, Zhang Y, et al. Effect of an Inactivated
Vaccine Against SARS-CoV-2 on Safety and Immunogenicity Outcomes: Interim Analysis of 2 Randomized Clinical Trials. JAMA, 2020,324(10):951960

10 Folegatti PM, Ewer KJ, Aley PK, et al. Safety and immunogenicity of the ChAdOx1 nCoV-19 vaccine against SARS-CoV-2: a preliminary report of a phase $1 / 2$, single-blind, randomised controlled trial. Lancet, 2020,396(10249):467-478

11 Wang F, Kream RM, Stefano GB. An Evidence Based Perspective on mRNA-SARS-CoV-2 Vaccine Development. Med Sci Monit, 2020,26:e924700

12 Zhu FC, Guan XH, Li YH, et al. Immunogenicity and safety of a recombinant adenovirus type 5-vectored COVID-19 vaccine in healthy adults aged 18 years or older: a randomised, double blind, placebo-controlled, phase 2 trial. Lancet, 2020,396(10249):479-488

13 COVID-19 vaccine tracker and landscape. Available from:https://www.who.int/publications/m/item/draftlandscape-of-covid-19-candidate-vaccines.[Accessed August 31, 2021].

14 Baden LR, El Sahly HM, Essink B, et al. Efficacy and Safety of the mRNA-1273 SARS-CoV-2 Vaccine. N Engl J Med, 2021,384(5):403-416

15 Polack FP, Thomas SJ, Kitchin N, et al. Safety and Efficacy of the BNT162b2 mRNA Covid-19 Vaccine. N Engl J Med, 2020,383(27):2603-2615

16 Voysey M, Clemens SAC, Madhi SA, et al. Safety and efficacy of the ChAdOx1 nCoV-19 vaccine (AZD1222) against SARS-CoV-2: an interim analysis of four randomised controlled trials in 8 Brazil, South Africa, and the UK. Lancet, 2021,397(10269):99-111

17 Xia S, Zhang Y, Wang Y, et al. Safety and immunogenicity of an inactivated SARS-CoV-2 vaccine, BBIBP-CorV: a randomised, double-blind, placebo-controlled, phase 1/2 trial. Lancet Infect Dis, 2021,21(1):39-51

18 Callaway E. Mix-and-match COVID vaccines trigger potent immune response. Nature, 2021,593(7860):491491

19 Hou H, Wang T, Zhang B, et al. Detection of IgM and IgG antibodies in patients with coronavirus disease 2019. Clin Transl Immunology, 2020,9(5):e01136

20 Doroftei B, Ciobica A, Ilie O-D, et al. Mini-Review Discussing the Reliability and Efficiency of COVID-19 Vaccines. Diagnostics (Basel), 2021,11(4):579

21 Salvagno GL, Henry BM, Piazza G di, et al. Anti-SARSCoV-2 Receptor-Binding Domain Total Antibodies Response in Seropositive and Seronegative Healthcare Workers Undergoing COVID2019 mRNA BNT162b2 Vaccination. Diagnostics (Basel), 2021,11(5):832

22 Terpos E, Trougakos IP, Apostolakou F, et al. Age-dependent and gender-dependent antibody responses against SARS-CoV-2 in health workers and octogenarians after vaccination with the BNT162b2 mRNA vaccine. Am J Hematol, 2021,96(7):E257-E259

23 Abu Jabal K, Ben-Amram H, Beiruti K, et al. Impact of age, ethnicity, sex and prior infection status on immunogenicity following a single dose of the BNT162b2 mRNA COVID-19 vaccine: real-world evidence from healthcare workers, Israel, December 2020 to January 2021. Euro Surveill, 2021,26(6):2100096

24 Grupper A, Sharon N, Finn T, et al. Humoral Response 
to the Pfizer BNT162b2 Vaccine in Patients Undergoing Maintenance Hemodialysis. Clin J Am Soc Nephrol, 2021,16(7):103701042

25 Ward H, Cooke G, Whitaker M, et al. REACT-2 Round 5: increasing prevalence of SARS-CoV-2 antibodies demonstrate impact of the second wave and of vaccine roll-out in England. Available from: https://www. medrxiv.org/content/10.1101/2021.02.26.21252512v1.

26 Jara A, Undurraga EA, González C, et al. Effectiveness of an Inactivated SARS-CoV-2 Vaccine in Chile. N Engl J Med, 2021,385(10):875-884

27 Murin CD, Wilson IA, Ward AB. Antibody responses to viral infections: a structural perspective across three different enveloped viruses. Nat Microbiol, 2019,4(5):734-747

28 Racine R, Winslow GM. IgM in microbial infections: taken for granted? Immunol Lett, 2009,125(2):79-85

29 Jalkanen P, Kolehmainen P, Häkkinen HK, et al. COVID-19 mRNA vaccine induced antibody responses against three SARS-CoV-2 variants. Nat Commun, 2021,12(1):3991

30 Röltgen K, Nielsen SCA, Arunachalam PS, et al. mRNA vaccination compared to infection elicits an IgG-predominant response with greater SARS-CoV-2 specificity and similar decrease in variant spike recognition. medRxiv. 2021:2021.04.05.21254952

31 Baden LR, El Sahly HM, Essink B, et al. Efficacy and Safety of the mRNA-1273 SARS-CoV-2 Vaccine. N Engl J Med, 2021,384(5):403-416

32 Omer SB, Yildirim I, Forman HP. Herd Immunity and Implications for SARS-CoV-2 Control. JAMA, 2020,324(20):2095-2096

33 Immunization coverage. Available from: https://www. who.int/news16 room/fact-sheets/detail/immunization- coverage. [Accessed October 2, 2021].

34 Mallory ML, Lindesmith LC, Baric RS. Vaccinationinduced herd immunity: Successes and challenges. J Allergy Clin Immunol, 2018,142(1):64-66

35 Kwok KO,LaiF, Wei WI, et al.Herd immunity-estimating the level required to halt the COVID-19 epidemics in affected countries. J Infect, 2020,80(6):e32-e33

36 Dörschug A, Frickmann H, Schwanbeck J, et al. Comparative Assessment of Sera from Individuals after S-Gene RNA-Based SARS-CoV-2 Vaccination with Spike-Protein-Based and Nucleocapsid Based Serological Assays. Diagnostics (Basel), 2021,11(3):426

37 Ranzani OT, Hitchings MDT, Dorion M, et al. Effectiveness of the CoronaVac vaccine in older adults during a gamma variant associated epidemic of covid-19 in Brazil: test negative case-control study. BMJ, 2021,374:n2015

38 Müller L, Andrée M, Moskorz W, et al. Age-dependent immune response to the Biontech/Pfizer BNT162b2 COVID-19 vaccination. Clin Infect Dis, 2021:ciab381

39 Quast I, Tarlinton D. B cell memory: understanding COVID-19. Immunity, 2021, 54(2):205-210

40 Khodadadi L, Cheng Q, Radbruch A, et al. The Maintenance of Memory Plasma Cells. Front Immunol, 2019,10:721

41 Baumgarth N, Nikolich-Žugich J, Lee FEH, et al. Antibody Responses to SARS-CoV33 2: Let's Stick to Known Knowns. J Immunol, 2020,205(9):2342-2350

42 Turner JS, O'Halloran JA, Kalaidina E, et al. SARSCoV-2 mRNA vaccines induce persistent human germinal centre responses. Nature, 2021,596(7870):109113

(Received Sep. 7, 2021; accepted Oct. 13, 2021) 